\title{
Dense and Symmetric Graph Formulation and Generation for Wireless Information Networks
}

\author{
Jaewook Yu, Dongsoo Kim, K. Wendy Tang \\ Department of Electrical and Computer Engineering \\ State University of New York at Stony Brook \\ Stony Brook 11794, USA \\ Email: $\{$ jaewook.yu|dongsoo.kim|wendy.tang\} estonybrook. edu
}

\begin{abstract}
Dense and symmetric graphs are useful for modeling fast information distribution in wireless information networks. In this paper, we focus on a specific family of dense and symmetric graphs, the Borel Cayley graphs. More specifically, we investigate the various parameters in the original formulation of Borel Cayley graphs defined in the matrix domain. By eliminating redundant parameters, we propose a new and simpler formulation of Borel Cayley graphs. This new formulation is defined in the integer domain and the group operation resembles the generation of pseudorandom numbers, hence the name pseudo-random formulation. Through the establishment of propositions and corollaries, we proved that certain parameters do not affect the diameter under a specific condition. This result provides a guideline in choosing appropriate generators and thus reducing the computation time in the search of good or bad generators. Using this new formulation, we also show that Borel Cayley graphs are isomorphic to a sub-class of Cayley graphs proposed by Dinneen. Finally, some guidelines for choosing generators to avoid disconnected graphs are also provided.
\end{abstract}

Keywords-Cayley graph, pseudo random graph, information networks.

\section{INTRODUCTION}

In designing a wireless information network, the network topology is modeled by graphs. The vertices of the graph correspond to wireless network nodes, and the edges correspond to wireless communication channels. Since present technology readily permits two-way communication, the graphs considered in this paper are undirected. In graph terminology, a regular graph is one that has the same number of edges at every vertex; the degree $\delta$ of a regular graph is the uniform number of connecting edges at every vertex; and the diameter, $D$, of a graph is the maximum of the set of minimum distances between any two vertices. Due to limited number of communication channels per network node, we are interested in regular graphs with a small degree. In particular, we are interested in dense graphs, that is, those having a large number of vertices for a given diameter and degree. The so called Moore bound provides an upper bound for the number of possible vertices present for a fixed $D$ and $\delta$. Graphs attaining these bounds are known as Moore graphs. However, Moore graphs have been proved to be non-existent except for diameter $D=2$ and $\delta=3,7$ and possibly 57 [1].

In addition to density, vertex-transitivity is a desirable attribute for efficient interconnection networks. Mathematically, a graph is vertex-transitive if for any two vertices $u$ and $v$, there is an automorphism that maps $u$ to $v$. Informally, this means the graph looks the same from any vertex. Such node symmetry also allows the use of identical processing/communicating elements at every vertex incorporating identical routing algorithms.

There is an increasing interest in a special class of graphs based on group theory, known as Cayley graphs. Basically, a Cayley graph is constructed from a finite group. The vertices of the graph are the elements of the group. Connections between vertices are defined by the group operation and a set of generators. There is no restriction in the choice of the underlying group. We can construct a Cayley graph over an arbitrary finite group and hence there are many varieties of Cayley graphs. It is known that all Cayley graphs are vertex-transitive [2]. This construction leads to many dense graphs and it has motivated several research efforts [3]-[7]. The attractiveness of Cayley graphs was further enhanced when Chudnovsky et. al discovered that certain Borel Cayley graphs, i.e., Cayley graphs based on Borel subgroups, are the densest degree4 , non-random graphs known for an interesting range of diameters [8].

In earlier reports [9]-[11], we have studied various properties of Cayley graphs, including representations and routing of these graphs. We have proved that all Cayley and Borel Cayley graphs can be transformed into Generalized Chordal Rings/Chordal Rings (GCR/CR) defined in the integer domain. Several time and space-efficient routing algorithms were also devised.

Furthermore, our previous results in [12] showed that the information distribution performance of Borel Cayley graphs is far superior than that of other well-known graph families. For example, the Borel Cayley graphs yielded a 2 to 100 times faster convergence than the small-world network (rewiring probability $p=0.01,0.1$ and 0.2 ). More importantly, the results also confirmed that Borel Cayley graph has the best scalability over a wide range of graph sizes and degrees [12].

In this paper we concentrate on the formulation and generation of dense Borel Cayley graphs. Through the establishment of propositions and corollaries, we propose a new and simpler formulation for Borel Cayley graphs. This new formulation resembles the generation of pseudorandom numbers. Furthermore, with this formulation, we show that Borel Cayley graphs are isomorphic to a subclass of Cayley graphs proposed by Dinneen using semi- 
direct product of groups [7]. Finally, some guidelines for choosing generators to avoid disconnected graphs are also provided.

\section{REVIEW}

Cayley graphs were first constructed by A. Cayley, a mathematician, in 1878 [2]. The construction of these graphs is described by finite algebraic group theory. A group $(\mathbf{V}, *)$ consists of a set $\mathbf{V}$ that is closed under inversion and a single law of composition $*$, also known as the group multiplication. There also exists an identity element $I \in \mathbf{V}$. A group is finite if there is finite number of elements in $\mathbf{V}$. In this section we review the definitions of Cayley graphs, Borel Cayley graphs and Cayley graphs proposed by Dinneen [7].

Definition 1 A graph $\mathbf{C}=(\mathbf{V}, \mathbf{G})$ is a Cayley graph with vertex set $\mathbf{V}$ if two vertices $v_{1}, v_{2} \in \mathbf{V}$ are adjacent $\Leftrightarrow$ $v_{1}=v_{2} * g$ for some $g \in \mathbf{G}$ where $(\mathbf{V}, *)$ is a finite group and $\mathbf{G} \subset \mathbf{V} \backslash\{I\} . \mathbf{G}$ is called the generator set of the graph.

Note that the identity element $I$ is excluded from G. This prevents the graph from having self-loops. A Cayley graph is undirected if $\mathbf{G}$ is closed under inversion, and the graph's degree is $|\mathbf{G}|$. In our research, we are interested in undirected, degree-four Cayley graphs mainly because current technology allows four-neighbor connections. In other words, we are concerned with Cayley graphs whose generator set consists of two group elements and their inverses.

The definition of a Cayley graph requires vertices to be elements of a group but does not specify a particular group. A family of Cayley graphs that includes some of the densest degree 4 graphs are formed from a subgroup, the Borel subgroup $\mathbf{B L}_{2}\left(\mathbf{Z}_{p}\right)$, of the general linear $2 \times 2$ matrices $\mathbf{G L}_{2}\left(\mathbf{Z}_{p}\right)$. The definition of the Borel subgroup is:

Definition 2 If $\mathbf{V}$ is a Borel subgroup, $\mathbf{B L}_{2}\left(\mathbf{Z}_{p}\right)$, of $\mathbf{G L}_{2}\left(\mathbf{Z}_{p}\right)$ with a parameter $a, a \in \mathbf{Z}_{p} \backslash\{0,1\}$, then

$$
\mathbf{V}=\left\{\left(\begin{array}{ll}
x & y \\
0 & 1
\end{array}\right): x=a^{t}(\bmod p), y \in \mathbf{Z}_{p}, t \in \mathbf{Z}_{k}\right\}
$$

where $p$ is prime and $k$ is the smallest positive integer such that $a^{k}=1(\bmod p)$.

The vertices of Borel Cayley graphs are $2 \times 2$ matrices that satisfy the definition of Borel subgroup, and modular $p$ matrix multiplication is chosen as the group operation *. Note that $N=|\mathbf{V}|=p \times k$, where $k$ is a factor of $p-1$ and $p$ is a prime number. By choosing specific generators, Chudnovsky et al. [8] produced Borel Cayley graphs that are the densest, nonrandom degree 4 graphs currently known for diameters 7 to 13 [8]. Table 1 compares the size of these graphs with that of the known graphs and the Moore bounds. It is clear that these Borel Cayley graphs show significant improvements in density. However, many questions about these graphs are not addressed in [8]. Most importantly, the question of how the choice of parameters contributes to improvements in density. Motivated by this question, our research focuses on Borel Cayley graphs and this paper presents some of our findings. It is also worth noting that the Borel Cayley graph discovered by Chudnovsky with $D=11, \delta=4$ has $n=38,764$. In our research, we have discovered yet another denser Borel Cayley graph with $n=41,831$ for $D=11, \delta=4$.

In a separate research effort, Dinneen proposed a Cayley graph constructed over a semi-direct product group [7]. The definition of this group is summarized as follows:

Definition 3 Given two cyclic groups $\mathbf{Z}_{m}$ and $\mathbf{Z}_{n}$, the semi-direct product group $\mathbf{S G}=\mathbf{Z}_{m} \times{ }_{\sigma} \mathbf{Z}_{n}$ is defined by a homomorphism $\sigma: \mathbf{Z}_{m} \rightarrow \operatorname{Aut}\left(\mathbf{Z}_{n}\right)$. Let an element $r$ be chosen from the group of units $\mathbf{U}\left(\mathbf{Z}_{n}\right)$. Define a mapping $\sigma^{\prime}(k)=\left(r^{c}\right)^{k}=r^{c k}$ where $c$ is chosen such that $r^{c m}=1$. The group $\mathbf{S G}$ has its multiplication table defined by

$$
\begin{aligned}
& \left(a_{0}, a_{1}\right) *_{\sigma}\left(b_{0}, b_{1}\right) \\
& \quad=\left(a_{0}+b_{0}(\bmod m), a_{1}+\sigma^{\prime}\left(a_{0}\right) b_{1}(\bmod n)\right) .
\end{aligned}
$$

\section{Parameters of Degree-4 Borel Cayley GRAPHS}

For ease of description, we assume a size $N$, degree- 4 Borel Cayley graph with generators $\mathbf{A}, \mathbf{B}, \mathbf{A}^{-1}$, and $\mathbf{B}^{-1}$. Furthermore,

$$
\begin{array}{ll}
\mathbf{A}=\left(\begin{array}{cc}
a^{t_{1}} & y_{1} \\
0 & 1
\end{array}\right), & \mathbf{A}^{-1}=\left(\begin{array}{cc}
a^{k-t_{1}} & <-a^{k-t_{1}} y_{1}>_{p} \\
0 & 1
\end{array}\right), \\
\mathbf{B}=\left(\begin{array}{cc}
a^{t_{2}} & y_{2} \\
0 & 1
\end{array}\right), & \mathbf{B}^{-1}=\left(\begin{array}{cc}
a^{k-t_{2}} & <-a^{k-t_{2}} y_{2}>_{p} \\
0 & 1
\end{array}\right),
\end{array}
$$

where $\langle x\rangle_{p}$ denotes $x \bmod p$. We note that $N=p \times k$ and the parameters: $p, a, k, t_{1}, t_{2}, y_{1}$ and $y_{2}$ are needed to specify a particular Borel Cayley graph. Among these parameters, $p, a, k$, related by $a^{k}=1(\bmod p)$, are responsible for the determination of a Borel group (Definition 2). However, connections and hence the diameter are determined by the generators $\mathbf{A}, \mathbf{B}$ and their inverses, characterized by $t_{1}, t_{2}, y_{1}$, and $y_{2}$. In our research effort, we investigate how these parameters affect each other and the diameter of the graph. Table 2 illustrates the variations in diameter $D$ as a result of different parameter values. In particular, the choices of $t_{1}$ and $t_{2}$ have a significant effect on the diameter $D$. For instance, a graph with size $N=1081$ have diameters ranging from 7 to 9 , depending on $t_{1}$ and $t_{2}$. In the following sections, we summarize our results.

\section{A. Parameters: $p$, $a$ and $k$}

As stated before, $N=p \times k$. That is, the size of a graph is determined by $p$ and $k$. Furthermore, $k$ is the order of $a(\bmod p)$, which implies that $k$ divides $p-1$. However the reason to choose a particular value of $a$ is not clear. Particularly, we have the following questions: (1) Is $k$ the smallest or largest order for all possible $a$ ? (2) How many 
TABle I: PARAMETERS OF BOREL CAYLEy GRAPHS

\begin{tabular}{ccccccccc}
\hline$N$ & $p$ & $k$ & $a$ & $t_{1}$ & $t_{2}$ & $y_{1}$ & $y_{2}$ & $D$ \\
\hline 1081 & 47 & 23 & 2 & 1 & 7 & 0 & 1 & 7 \\
& & & & 2 & 10 & 0 & 1 & 7 \\
& & & & 3 & 8 & 0 & 1 & 8 \\
\hline 2943 & 109 & 27 & 7 & 1 & 6 & 0 & 1 & 8 \\
\hline 7439 & 173 & 43 & 16 & 4 & 10 & 0 & 1 & 9 \\
\hline 15657 & 307 & 51 & 4 & 2 & 16 & 0 & 1 & 10 \\
& & & & 2 & 12 & 0 & 1 & 10 \\
& & & & 4 & 4 & 0 & 1 & 13 \\
& & & & 1 & 2 & 0 & 1 & 12 \\
\hline 82901 & 911 & 91 & 2 & 31 & 34 & 0 & 1 & 12 \\
\hline
\end{tabular}

$a$ have order $k$ ? (3) For those $a$ of the same order, do they generate the same set of numbers? In this section, we address these questions.

For any element $a \in \mathbf{Z}_{p}$, the smallest order is always 1 , when $a=1$; and the biggest order is always $p-1$, when $a$ is a primitive root of $p$. Furthermore, the possible values of $k$ are the factors of $p-1$. For example, when $p=47$, $p-1=2 \times 23$, and the possible values of $k$ are $1,2,23$ and 46 .

The number of $a$ with order $k$ is given by the Euler function $\phi(k)$. Furthermore, these $a$ generate the same set of numbers. These observations are supported by existing theorems [13]. They are summarized as follows:

Theorem 1 The number of $a$ with order $k$ is $\phi(k)$, where $\phi()$ is the Euler function. That is, for $k=p_{1}^{c_{1}} \times p_{2}^{c_{2}} \times$ $p_{3}^{c_{3}} \times \cdots$

$\phi(k)=k \times\left(1-1 / p_{1}\right) \times\left(1-1 / p_{2}\right) \times\left(1-1 / p_{3}\right) \times \cdots$,

where $p_{1}, p_{2}, p_{3}, \cdots$ are prime numbers.

Theorem 2 For a prime $p$, if $k$ divides $p-1$, then $x^{k}=1$ $(\bmod p)$ has exactly $k$ roots.

The fact that different a generate the same set of numbers, implies that the choice of $a$ has no effect on the group or the graph. Once $p$ and $k$ are being fixed, the size of the graph is determined, and any $a$ with order $k$ can be chosen.

\section{B. Parameters: $t_{1}, t_{2}, y_{1}$ and $y_{2}$}

The parameters $t_{1}, t_{2}, y_{1}$ and $y_{2}$ define the generators $\mathbf{A}, \mathbf{B}, \mathbf{A}^{-1}$ and $\mathbf{B}^{-1}$, which in turn define the connections and hence the diameter of the graph. From Table 2, it is clear that the choices of $t_{1}$ and $t_{2}$ play a crucial part in the determination of diameter. Furthermore, our computer analysis indicates that changing $y_{1}$ and $y_{2}$ do not change the diameter. This empirical observation is verified through the establishment of the following propositions. Again, we assume the generators of the degree- 4 Borel Cayley graphs are $\mathbf{A}, \mathbf{B}, \mathbf{A}^{-1}$ and $\mathbf{B}^{-1}$, according to Equation (2).
Proposition $1\left(1-a^{t_{2}}\right) y_{1}=\left(1-a^{t_{1}}\right) y_{2} \quad(\bmod p) \Leftrightarrow$ $\mathbf{A B}=\mathbf{B A}$

The proof of this proposition is a straight forward substitution and is omitted.

Proposition 2 For any paths $\mathrm{X}, \mathrm{Y}$, composed of generators $\mathbf{A}, \mathbf{B}, \mathbf{A}^{-1}$ and $\mathbf{B}^{-1}$, let

$$
\begin{aligned}
& \mathbf{X}=\left(\begin{array}{cc}
a^{<i t_{1}+j t_{2}>_{k}} & <g y_{1}+h y_{2}>_{p} \\
0 & 1
\end{array}\right) \text { and } \\
& \mathbf{Y}=\left(\begin{array}{cc}
a^{<i^{\prime} t_{1}+j^{\prime} t_{2}>_{k}} & <g^{\prime} y_{1}+h^{\prime} y_{2}>_{p} \\
0 & 1
\end{array}\right)
\end{aligned}
$$

where $<x>_{p}$ denotes $x(\bmod p)$. Then

$$
\begin{aligned}
& \mathbf{X}=\mathbf{Y} \\
\Leftrightarrow & i t_{1}+j t_{2}=i^{\prime} t_{1}+j^{\prime} t_{2}(\bmod k) \text { and } \\
& \left\{\begin{array}{l}
g=g^{\prime} \text { and } h=h^{\prime}(\bmod p) \text { or } \\
\left(1-a^{t_{2}}\right) y_{1}=\left(1-a^{t_{1}}\right) y_{2}(\bmod p) .
\end{array}\right.
\end{aligned}
$$

The proof of this proposition is included in [10] and is not repeated here. From this result, if $\mathbf{A B} \neq \mathbf{B A}$,

$$
\mathbf{X}=\mathbf{Y} \Leftrightarrow\left\{\begin{array}{l}
i t_{1}+j t_{2}=i^{\prime} t_{1}+j^{\prime} t_{2}(\bmod k) \text { and } \\
g=g^{\prime} \text { and } h=h^{\prime}(\bmod p) .
\end{array}\right.
$$

The determination of the diameter of a graph basically involves generating the entire set of vertices from different compositions of generators. Equation (3) shows that, whether two different compositions, $\mathbf{X}$ and $\mathbf{Y}$ correspond to the same node is independent of the values of $y_{1}$ and $y_{2}$. In other words, we have a useful corollary:

Corollary 1 The values of $y_{1}$ and $y_{2}$ do not affect the diameter, iff $\mathbf{A B} \neq \mathbf{B A}$.

\section{A PSEUdo-RANDOM Formulation}

In Section III-A and III-B, we have shown that the choices of $a, y_{1}$ and $y_{2}$ do not affect the connections of a Borel Cayley graph. The parameters that determine a Borel Cayley graph are: $p, k, t_{1}, t_{2}$. Based on this finding, we can use a constrained, simpler formulation of a Borel group. Assume a Borel group as defined in Definition 2. We define a Borel coordinate group $\mathbf{B}_{p, k}$ as follows:

Definition 4 For any prime number $p$ and a factor of $p-1$, $k$, choose any $a$ such that $a^{k}=1(\bmod p)$. We have a $\mathbf{B}_{p, k}$ with size $N=p \times k$ and

$$
\mathbf{B}_{p, k}=\left\{(t, y): t \in \mathbf{Z}_{k}, y \in \mathbf{Z}_{p}\right\} .
$$

For any $(t, y),\left(t^{\prime}, y^{\prime}\right) \in \mathbf{B}_{p, k}$, the group operation $*$ is defined as:

$$
(t, y) *\left(t^{\prime}, y^{\prime}\right)=\left(<t+t^{\prime}>_{k},<a^{t} y^{\prime}+y>_{p}\right) .
$$

Accordingly, the generators $\mathbf{A}$ and $\mathbf{B}$ in the group can be defined as:

$$
\begin{aligned}
& \mathbf{A}=\left(t_{1}, y_{1}\right), \mathbf{A}^{-1}=\left(k-t_{1},<-a^{k-t_{1}} y_{1}>_{p}\right) \\
& \mathbf{B}=\left(t_{2}, y_{2}\right), \mathbf{B}^{-1}=\left(k-t_{2},<-a^{k-t_{2}} y_{2}>_{p}\right) .
\end{aligned}
$$


Since $y_{1}$ and $y_{2}$ do not affect the diameter, the simplest choices for $y_{1}$ and $y_{2}$ are

$$
\begin{aligned}
& y_{1}=\left\{\begin{array}{l}
0 \text { if } t_{1} \neq 0 \\
1 \text { if } t_{1}=0
\end{array}\right. \text { and } \\
& y_{2}=\left\{\begin{array}{ll}
0 & \text { if } t_{2} \neq 0 \text { and } y_{1} \neq 0 \\
1 & \text { if } t_{2}=0 \text { or } y_{1}=0
\end{array} .\right.
\end{aligned}
$$

Basically this new formulation has eliminated less essential parameters and retained the properties of the original group. In this new formulation, only two integers are needed to specify an element; while in the original group, an element is represented by a $2 \times 2$ matrix, which requires four integers to specify. However, we observe that the elements on the second row of a Borel matrix are always 0 and 1 , which implies that such a formulation carries redundant information. In our new formulation, modular integer arithmetic has replaced the more complicated, modular matrix multiplication of the original group.

Furthermore, the new group operation $*$ (Equation (4)) resembles the generation of pseudo-random numbers. The generation of pseudo-random numbers by digital computers has been well studied. The almost universally used method is the mixed congruential scheme, given by

$$
x_{i+1}=\lambda x_{i}+c \quad(\bmod T)
$$

where $\lambda$ and $c$ are fixed odd integers and the $x_{i}<T$ are the sequence of random numbers. We observed that the operation on the $y$-coordinate in Equation (4) is similar to the mixed congruential scheme, Equation (7). Because of such similarity, we called this new formulation, a pseudorandom formulation of Borel Cayley graphs.

In comparing Equations (4) and (1), we also found a striking resemblance. Indeed, the Borel coordinate group, $\mathbf{B}_{p, k}$, defined in Definition 4 is a sub-class of the semidirect product group SG proposed by Dinneen and defined in Definition 3. More specifically, if we choose, $m=$ $k, n=p, r^{c}=a$ (Definition 3), $\mathbf{S G}=\mathbf{Z}_{m} \times{ }_{\sigma} \mathbf{Z}_{k}=\mathbf{B}_{p, k}$, where $a$ has order $k$ in $\mathbf{Z}_{p}$. It is therefore not surprising that the densest known degree-4 Cayley graphs provided in [7] have the same number of nodes as that of Table 1. Using a computer program, we have also verified that the generators listed in [7] produce the same diameter in our pseudo-random formulation of the corresponding Borel Cayley graph.

\section{CHOICE OF GENERATOR PAIRS}

The definition of Borel Cayley graphs shows the connection of the graphs is governed by the choice of generators $\left(t_{1}, y_{1}\right)$ and $\left(t_{2}, y_{2}\right)$. In fact, we observed that most generator pairs yield densely connected graphs within a narrow range of diameters. However, it is also observed that a small portion of generator pairs gives rise to disconnected graphs which are not desirable in a wireless information network. For example, most networked systems such as computer networks, wireless communication networks, and cooperative vehicles require the underlying network topology to have strong connectivity and without
TABLE II: SIZE AND CORRESPONDING PARAMETERS OF THE BOREL CAYLEY GRAPHS.

\begin{tabular}{cccccc}
\hline set & $N$ & $p$ & $k$ & $a$ & samples \\
\hline & 1081 & 47 & 23 & 2 & 45 \\
& 2265 & 151 & 15 & 2 & 45 \\
A & 3081 & 79 & 39 & 2 & 45 \\
& 4112 & 257 & 16 & 2 & 45 \\
& 5253 & 103 & 51 & 2 & 45 \\
\hline \multirow{2}{*}{ B } & 2211 & 67 & 33 & 6 & 45 \\
& 4063 & 239 & 17 & 6 & 45 \\
\hline
\end{tabular}

any isolated node. Thus, it is critical to avoid generating disconnected graphs by choosing appropriate parameters for the generators. This section summarizes the patterns of various parameters that will lead to disconnected Borel Cayley graphs and therefore should be avoided.

\section{A. Simulation Parameters}

According to Section IV, we only consider generators $A=\left(\begin{array}{cc}a^{t_{1}} & 0 \\ 0 & 1\end{array}\right), B=\left(\begin{array}{cc}a^{t_{2}} & 1 \\ 0 & 1\end{array}\right)$ and their inverses $A^{-1}$, $B^{-1}$. The parameters $y_{1}$ and $y_{2}$ are chosen according to Equation (6). In addition, we set the range of $t_{1}$ and $t_{2}$ to $1 \leq t_{1}<t_{2} \leq 10$ instead of using all $\left(t_{1}, t_{2}\right)$ pairs in the range $0 \leq t_{1}<t_{2} \leq(k-1)$. Thus, the total number of generator combinations is 45 where each $\left(t_{1}, t_{2}\right)$ pair generates a unique Borel Cayley graph with different connectivity pattern. We concede, however, that these limited choices imply that our conjectured guidelines need further verification by sampling the entire possible space of $t_{1}$ and $t_{2}$. This paper is a work-in-progress report on the status of our findings and we plan to perform more simulations to further confirm our results as will be discussed in the Conclusion section.

Table II summarizes the parameters of Borel Cayley graphs used in our experiments. There are two sets of graphs. The simulation set A generates 1081, 2265, 3081, 4112 and 5253 nodes Borel Cayley graphs while the simulation set B generates 2211 and 4063 nodes graphs. We use the data from the simulation set $\mathrm{A}$ to find patterns of good or bad generator pairs. In addition, the simulation set $\mathrm{B}$ is designed to validate our observations.

For each of the generated Borel Cayley graph, we also computed its algebraic connectivity, the second smallest eigenvalue of the graph Laplacian. It is well known that the algebraic connectivity of a graph is larger than 0 if and only if the graph is connected. The theoretical foundation of algebraic connectivity has been established by $M$. Fiedler in [14] and is not repeated here.

\section{B. Patterns of bad generators}

Based on the $45\left(t_{1}, t_{2}\right)$ generator pairs and their corresponding graphs for each of 1081-, 2265-, 3081-, 4112- and 5253-node Borel Cayley graphs, we found that there exists a pattern of good or bad generators.

The algebraic connectivity results of the generated graphs revealed that most of the Borel Cayley graphs generated were connected graphs while a smaller portion is disconnected. More specifically, 4, 3, 10 and 4 out of the 
TABLE III: GENERATOR PARIS OF DISCONNECTED GRAPHS FROM THE THE SIMULATION SET A

\begin{tabular}{|c|c|c|c|c|c|}
\hline$N$ & $p$ & $k$ & $\mathbf{d}(k)$ & generator $\left(t_{1}, t_{2}\right)$ & $\mathbf{c d}\left(t_{1}, t_{2}\right)$ \\
\hline 1081 & 47 & 23 & 23 & - & - \\
\hline 2265 & 151 & 15 & 3,5 & $\begin{array}{l}(3,6),(3,9) \\
(5,10) \\
(6,9)\end{array}$ & $\begin{array}{l}3 \\
5 \\
3\end{array}$ \\
\hline 3081 & 79 & 39 & 3,13 & $\begin{array}{l}(3,6),(3,9) \\
(6,9)\end{array}$ & $\begin{array}{l}3 \\
3\end{array}$ \\
\hline 4112 & 257 & 16 & 2 & $\begin{array}{l}(2,4),(2,6),(2,8),(2,10) \\
(4,6),(4,8),(4,10) \\
(6,8),(6,10) \\
(8,10)\end{array}$ & $\begin{array}{l}2 \\
2 \\
2 \\
2\end{array}$ \\
\hline 5253 & 103 & 51 & 3,17 & $\begin{array}{l}(3,6),(3,9) \\
(6,9)\end{array}$ & $\begin{array}{l}3 \\
3\end{array}$ \\
\hline
\end{tabular}

45 samples for 2265-, 3081-, 4112- and 5253-node Borel Cayley graphs, respectively, were disconnected graphs. On the other hand, there was no disconnected graph generated in 1081-node case. We are interested in finding out if there is any pattern of generator pairs that result in disconnected graphs.

In Table III, we summarized the parameters of the disconnected Borel Cayley graphs, $p, k$ and the generator pair $\left(t_{1}, t_{2}\right)$ in addition to the divisors of $k$ denoted by $\mathbf{d}(k)$ and the common devisors of $t_{1}$ and $t_{2}, \operatorname{cd}\left(t_{1}, t_{2}\right)^{1}$. Note that one of $k$ 's divisors always appears in the common divisors of $\left(t_{1}, t_{2}\right)$ pairs. Also, the opposite was always true. That is, if a Borel Cayley graph is disconnected, then $\mathbf{c d}\left(t_{1}, t_{2}\right)$ is always an element of the $\mathbf{d}(k)$ set. In fact, $100 \%$ of the generator pairs in Table III share their common divisors with the divisors of $k$. For example in 2265 -node case, the common divisors of $t_{1}$ and $t_{2}$ of all the disconnected graphs, 3 and 5 , are also the divisors of $k=15$. In addition, the common divisors of all the other generator pairs other than $(3,6),(3,9)$ and $(6,9)$ are not factors of $k$.

Furthermore, we also conjecture that the common divisor $\mathbf{c d}\left(t_{1}, t_{2}\right)$ is not a factor of $k$ if and only if the Borel Cayley graph is connected. For example, the parameter $k$ of 1081-node Borel Cayley graphs is the prime number 23 of which only two factors are trivial factor 1 and itself. The fact that the divisor 23 of $k$ is a prime number and that it is not within the range of $t_{1}$ or $t_{2}$ guarantees that no common divisor $\operatorname{cd}\left(t_{1}, t_{2}\right)$ divides $k$.

To support this conjecture, we further experimented with the simulation set B in Table II. First, in Table IV, the generator pairs of all disconnected 2211-node Borel Cayley graphs were the same as the ones of disconnected 3081- and 5253-node Borel Cayley graphs. The fact that the common divisors of disconnected graphs of all 2211 , 3081 and 5253 cases are 3 and it appears in the divisor set of $k$ supports our conjecture. Secondly, there is no disconnected graph generated for 4063-node Borel Cayley graphs in the range of $\left(t_{1}, t_{2}\right)$ pairs we considered. That is

${ }^{1}$ Of course, we do not consider trivial divisors 1 or parameter itself as $\mathbf{d}(k)$ or $\mathbf{c d}\left(t_{1}, t_{2}\right)$. For $\mathbf{d}(k)$, however, if the parameter $k$ itself is the one and only divisor, then we accept $k$ as a divisor of $k$.
TABLE IV: GENERATOR PARIS OF DISCONNECTED GRAPHS FROM THE SIMULATION SET B

\begin{tabular}{cccclc}
\hline size & $p$ & $k$ & $\mathbf{d}(k)$ & generator $\left(t_{1}, t_{2}\right)$ & $\mathbf{c d}\left(t_{1}, t_{2}\right)$ \\
\hline 2211 & \multirow{2}{*}{67} & \multirow{2}{*}{33} & 3,11 & $\begin{array}{l}(3,6),(3,9) \\
(6,9)\end{array}$ & $\begin{array}{l}3 \\
3\end{array}$ \\
\hline 4063 & 239 & 17 & 17 & - & - \\
\hline
\end{tabular}

because the only factor 17 of the parameter $k$ of 4063-node Borel Cayley graphs does not fall into the experimented range $1 \leq t_{1}<t_{2} \leq 10$ and hence, there is no generator pairs of which common divisors divide the factor of $k$.

\section{Guideline for choosing Borel Cayley graph parameters}

Based on our observations and conjectures from the previous section, we summarize some guidelines for choosing the parameter $k$ and the generator pairs $\left(t_{1}, t_{2}\right)$ that guarantees a connected Borel Cayley graph.

Odd $k$

For even number $k$, the divisor 2 of $k$ is a common divisor of all even $\left(t_{1}, t_{2}\right)=($ even, even $)$ pairs which means half of the generator pairs will result in disconnected graphs. By choosing odd number $k$, we can avoid such cases.

Prime $k$

If possible, choose a prime number for parameter $k$. Based on our conjecture, all generator pairs $\left(t_{1}, t_{2}\right)$ except for the cases that $\mathbf{c d}\left(t_{1}, t_{2}\right)$ divides $k$ will result in a connected graph when $k$ is prime

Given $k$, choose $\left(t_{1}, t_{2}\right)$ s.t. $\mathbf{c d}\left(t_{1}, t_{2}\right) \notin\{d \mid d=\mathbf{d}(k)\}$ Once the parameter $k$ is chosen, the generator $\left(t_{1}, t_{2}\right)$ has to be chosen such that any common divisor of $t_{1}$ and $t_{2}\left(\mathbf{c d}\left(t_{1}, t_{2}\right)\right)$ is not a divisor of $k$ denoted as $\mathbf{d}(k)$.

\section{CONCLUSIONS AND FUTUREWORKS}

Dense, symmetric graphs are used for modeling fast information distribution in wireless information networks. Applications can be found in consensus protocols [12] and MAC protocol designs [15].

Cayley graphs are attractive since they are symmetric and provide densest known degree- 4 graphs for a range of diameters [9]. These graphs are constructed over a group of matrices. Connections of the graph are defined by postmultiplying vertices with generators in the generator set. Appropriate choices of generators are critical to the diameter of the graph.

Despite the potential of using Borel Cayley graphs for fast distribution of information in wireless information network, little is known about the parameters of these graphs. Most importantly, the relationship between the generators and the diameter of the graph is unknown. Currently, identification of "good" generators are achieved through random or extensive systematic search of all possibilities [16]. In an effort to resolve this problem, we 
investigate the parameters of Borel Cayley graphs. This paper summarizes our findings.

By eliminating redundant information, we propose a new and simpler formulation of Borel Cayley graphs. This new formulation is defined in the integer domain and the group operation resembles the generation of pseudorandom numbers, hence the name pseudo-random formulation. In this new formulation, elements of the group are defined as coordinate pairs. For a degree- 4 Borel Cayley graph, the generators are now considered as $\mathbf{A}=\left(t_{1}, y_{1}\right)$ and $\mathbf{B}=\left(t_{2}, y_{2}\right)$.

Through the establishment of propositions and corollaries, we proved that the values of $y_{1}$ and $y_{2}$ do not affect the diameter if and only if $\mathbf{A B} \neq \mathbf{B A}$. This result provides a guideline in choosing appropriate generators and thus reducing the computation time in the search of "good" generators. Using this new formulation, we also show that Borel Cayley graphs are isomorphic to a subclass of Cayley graphs proposed by Dinneen [7].

Finally, through running and observing a range of extensive simulations, we conjectured a set of guidelines for choosing the appropriate parameters to avoid generating disconnected graphs. However, we concede that, the guidelines are established by sampling a limited space of the generator parameters. A more extensive search involving sampling the entire space of the generator set is needed to confirm these conjectures. We are currently working on this aspect of the project.

\section{ACKNOWLEDGEMENT}

The authors are partially supported by the National Science Foundation under Grant No. CNS 0829656. Any opinions, findings and conclusions or recommendations expressed in this article are those of the authors and do not necessarily reflect the views of the National Science Foundation.

We also would like to thank Dr. Eric Noel at AT\&T Labs Research for his contribution to this work. Mr. Jaewook $\mathrm{Yu}$ and Dongsoo Kim are being jointly supervised by Dr. Noel and Wendy Tang at Stony Brook University under an AT\&T and Stony Brook University collaborative agreement.

\section{REFERENCES}

[1] J. Bermond, C. Delorme, and J. Quisquater, "Strategies for interconnection networks: Some methods from graph theory," Journal of Parallel and Distributed Computing, vol. 3, no. 4, pp. 433-449, 1986.

[2] N. Biggs, Algebraic graph theory. Cambridge University Press, 1993.

[3] S. Akers and B. Krishnamurthy, "A group-theoretic model for symmetric interconnection networks," IEEE Trans. Comput., vol. 38, no. 4, pp. 555-566, 1989.

[4] G. Carlsson, J. Cruthirds, H. Sexton, and C. Wright, "Interconnection networks based on a generalization of cubeconnected cycles," IEEE Trans. Comput., vol. 100, no. 34, pp. 769-772, 1985.
[5] F. Annexstein, M. Baumslag, and A. Rosenberg, "Group action graphs and parallel architectures," SIAM Journal on Computing, vol. 19, pp. 544-569, 1990.

[6] K. Blaha, "Algorithms for permutation groups and Cayley networks," Ph.D. dissertation, University of Oregon, Eugene, OR, USA, 1989.

[7] M. J. Dinneen, "Algebraic methods for efficient network constructions," Master's thesis, Department of Computer Science, University of Victoria, Victoria, BC, Canada, 1991.

[8] D. Chudnovsky, G. Chudnovsky, and M. Denneau, "Regular graphs with small diameter as models for interconnection networks," in Proc. 3rd Internat. Conf. on Supercomputing ICS'88, 1988, pp. 232-239.

[9] K. W. Tang, "Dense, symmetric interconnection networks," Ph.D. dissertation, University of Rochester, Rochester, NY, USA, 1991.

[10] K. W. Tang and B. W. Arden, "Representations of Borel Cayley graphs," SIAM Journal on Discrete Mathematics, vol. 6, no. 4, pp. 655-676, 1993.

[11] B. W. Arden and K. W. Tang, "Representations and routing for Cayley graphs [computer networks]," IEEE Trans. Commun., vol. 39, no. 11, pp. 1533-1537, Nov 1991.

[12] J. Yu, E. Noel, and K. W. Tang, "Pseudo-random graphs for fast consensus protocol," in Proc. of the 2009 International Conference on Parallel and Distributed Processing Techniques and Applications (PDPTA'09), Jul.13-16, 2009.

[13] G. Hardy and E. Wright, An introduction to the theory of numbers. Oxford University Press, USA, 1979.

[14] M. Fiedler, "Algebraic Connectivity of Graphs," Czechoslovak Mathematical Journal, vol. 23, pp. 298-305, 1973.

[15] E. Noel and K. W. Tang, "Novel sensor MAC protocol applied to Cayley and manhattan street networks with CrossBow MICA2," in Proc. of the 3rd Annual IEEE Communications Society on Sensor and Ad Hoc Communications and Networks (SECON'06), vol. 2, Sep.25-28, 2006, pp. 626-631.

[16] L. Campbell, G. Carlsson, M. Dinneen, V. Faber, M. Fellows, M. Langston, J. Moore, A. Mullhaupt, and H. Sexton, "Small diameter symmetric networks from linear groups," IEEE Trans. Comput., vol. 41, no. 2, pp. 218-220, 1992. 